\title{
DESEMPENHO COMPARATIVO DE UM MOTOR DE CICLO DIESEL UTILIZANDO DIESEL E MISTURAS DE BIODIESEL ${ }^{1}$
}

\author{
Comparative performance of a cycle diesel engine using diesel and biodiesel mixtures
}

\author{
Ronald Leite Barbosa², Fábio Moreira da Silva³, Nilson Salvador ${ }^{4}$, Carlos Eduardo Silva Volpato
}

\begin{abstract}
RESUMO
Os atuais elevados preços do barril de petróleo no mercado internacional, a possibilidade de geração de postos de trabalho e renda com a conseqüente fixação do homem no campo, as excelentes e variadas condições climáticas e os tipos de relevo fazem com que o Brasil, com suas extensas áreas agricultáveis, destaque-se no cenário mundial em relação à sua grande potencialidade de geração de combustíveis alternativos. A situação ambiental faz com que o ser humano trabalhe no desenvolvimento de alternativas energéticas, destacando-se aquelas oriundas de fontes renováveis e biodegradáveis de caráter eminentemente sustentável. Assim, objetivou-se com este trabalho avaliar o desempenho de um motor ciclo diesel, funcionando em momentos distintos com diesel mineral e misturas deste com biodiesel nas proporções equivalentes a B2 (98\% de diesel mineral e 2\% de biodiesel), B5 (95\% de diesel mineral e 5\% de biodiesel), B20 (80\% de diesel mineral e 20\% de biodiesel) e B100 (100\% de biodiesel). Para a realização dos ensaios, foi utilizado um motor ciclo diesel de um trator VALMET $85 \mathrm{id}$, de 58,2kW (78 cv), de acordo com metodologia estabelecida pela norma NBR 5484 da ABNT (1985) que se refere ao ensaio dinamométrico de motores de ciclo Otto e Diesel. Concluiu-se que a potência do motor ao se utilizar biodiesel foi inferior àquela quando se utilizou diesel mineral. Observou-se que, em algumas rotações, as misturas B5 e B20 apresentaram potência igual ou até superior, em algumas situações, àquela quando se utilizou diesel mineral. A melhor eficiência térmica do motor foi verificada na rotação de $540 \mathrm{rpm}$ da TDP equivalente a $1720 \mathrm{rpm}$ do motor.
\end{abstract}

Termos para indexação: Biodiesel, desempenho, motor ciclo diesel, combustível alternativo.

\begin{abstract}
It is considered that, in a close future, the petroleum reservations economically viable will tend to the shortage. Besides it, the exacerbated current price levels of the petroleum barrel in the international market, the possibility of employment generation and income with the consequent fixation of man country life, the excellent and varied climatic conditions and several types of terrain become the country, with extensive workable areas, stand out in the world scenery if considering its great potentiality on generation of alternative fuels. The environmental preservation, important subject nowadays, makes that the human being work in searches for the development of alternative energies, mainly those originating from renewable and biodegradable sources of sustantable character. Taking in consideration those searches, the purpose of this work was to evaluate the performance of a diesel engine working in different moments with mineral diesel and mixtures of mineral diesel and biodiesel in the equivalent proportions B2 (98\% mineral diesel and 2\%biodiesel), B5 (95\% mineral diesel and 5\%biodiesel), B20 (80\% mineral diesel and 20\%biodiesel), and, finally, B100 (100\% biodiesel). The rehearsal was accomplished in the dependences of the Engineering Department at UFLA - Federal University of Lavras, in Lavras, Minas Gerais, in July, 2005. For the accomplishment of the rehearsals it, was used an engine cycle diesel of a tractor VALMET $85 \mathrm{id}$, of $58,2 \mathrm{~kW}(78 \mathrm{cv})$, following it methodology established by the norm NBR 5484 of ABNT (1985), that refers to the rehearsal dynamometric of engines cycle Otto and Diesel being proceeded. One noticed ended that the potency of the motor when using biodiesel was lower than one when using mineral diesel. One observed that, in some rotations, the mixtures B5 and B20 presented the same potency or even higher, in some situations, than the one when if using mineral diesel. The best thermal efficiency of the motor was verified in the rotation of $540 \mathrm{rpm}$ of equivalent TDP to $1720 \mathrm{rpm}$ of the motor.
\end{abstract}

Index terms: Biodiesel, performance of a diesel engine, alternative fuel.

(Recebido em 20 de novembro de 2006 e aprovado em 2 de maio de 2007)

\footnotetext{
${ }^{1}$ Parte da dissertação de mestrado apresentada à Universidade Federal de Lavras/UFLA, Departamento de Engenharia para obtenção do título de mestre.

²Engenheiro Agrícola, Mestre - Departamento de Mecânica - Centro Federal de Educação Tecnológica de Ouro Preto/CEFET - Avenida Michael Pereira de Souza, 3007 - 36415-000 - Campinho - Congonhas, MG - roleba@uai.com.br

${ }^{3}$ Doutor em Engenharia Mecânica, Professor Adjunto III - Departamento de Engenharia/DEG - Universidade Federal de Lavras/UFLA - Cx. P. 3037 37200-000 - Lavras, MG - famsilva@ufla.br

${ }^{4}$ Doutor em Engenharia Agrícola, Professor Adjunto IV - Departamento de Engenharia/DEG - Universidade Federal de Lavras/UFLA - Cx. P. 3037 37200-000 - Lavras, MG - salvador@ufla.br

${ }^{5}$ Doutor em Engenharia Agrícola, Professor Adjunto III - Departamento de Engenharia/DEG - Universidade Federal de Lavras/UFLA - Cx. P. 3037 37200-000 - Lavras, MG - volpato@ufla.br
} 


\section{INTRODUÇÃO}

Com a crise do petróleo nos anos 70 do século XX, o mundo despertou para a busca por formas alternativas de energia. No Brasil, a crise culminou com a criação do Próálcool nos anos 80 do século passado e outros programas de incentivo ao uso de combustíveis alternativos como óleos vegetais, gasogênio, gás natural, biogás, etc.

Segundo Grando (2005), a crescente demanda por combustíveis renováveis como alternativa aos combustíveis fósseis e a maior preocupação internacional com o aquecimento global, amplificada agora com a entrada em vigor do Protocolo de Kyoto, evidencia os benefícios dos biocombustíveis. O biodiesel contribuirá para melhorar as condições ambientais a partir da redução da emissão de gases poluentes.

O Biodiesel é um combustível renovável, biodegradável e ambientalmente correto, sucedâneo ao óleo diesel mineral, constituído de uma mistura de ésteres metílicos ou etílicos de ácidos graxos, obtidos da reação de transesterificação de qualquer triglicerídeo com um álcool de cadeia curta, metanol ou etanol, respectivamente (PARENTE, 2005).

De acordo com Oliveira \& Costa (2005), a implantação de um programa energético com biodiesel abre oportunidades para grandes benefícios sociais decorrentes do alto índice de geração de empregos por capital investido, culminando com a valorização do campo e a promoção do trabalhador rural, além das demandas por mão-de-obra qualificada para o processamento e, em muitos casos, beneficiamento do óleo vegetal. Salienta-se, entretanto, a reversão no fluxo internacional de capitais, uma vez que o aproveitamento do biodiesel permite uma redução das importações de diesel e a comercialização internacional de Certificados de Redução de Emissões de gases causadores do efeito estufa.

Como combustível, o biodiesel possui características vantajosas em relação aos combustíveis derivados do petróleo, podendo ser citada a condição de ser virtualmente livre de enxofre e de compostos aromáticos; alto número de cetano; teor médio de oxigênio; maior ponto de fulgor; menor emissão de partículas, $\mathrm{HC}$, $\mathrm{CO}$ e de $\mathrm{CO}_{2}$; caráter não tóxico e biodegradável, além de ser proveniente de fontes renováveis. Ele permite que se estabeleça um ciclo fechado de carbono no qual o $\mathrm{CO}_{2}$ é absorvido quando a planta cresce e é liberado quando o mesmo é queimado na combustão do motor. Estudo conjunto realizado pelos Departamentos de Energia e de Agricultura dos Estados Unidos mostra que o biodiesel reduz em $78 \%$ as emissões líquidas de $\mathrm{CO}_{2}$ (D'ARCE, 2005).

Desta forma, produzido a partir de fontes renováveis tais como óleos vegetais (girassol, amendoim, algodão, soja, mamona, etc.), gorduras animais e óleos de frituras, o biodiesel surge como uma opção de substituição do diesel mineral nos motores de combustão por compressão (PROGRAMA NACIONAL DE PRODUÇÃO E USO DE BIODIESEL, 2005).

A soja, única oleaginosa de grande disponibilidade no Brasil, com quase 60 milhões de toneladas colhidas este ano, oferece apenas $20 \%$ de óleo ao ser processada. Seria necessário ampliar em $20 \%$ a colheita nacional para produzir dois bilhões de litros de biodiesel que o país necessitará se adotar uma mistura de apenas 5\% (B5). Contudo, as fontes podem ser diversificadas e tornar rentável a pequena agricultura em muitas regiões, promovendo desenvolvimento e inclusão social. Além disso, o Brasil pode converter-se no grande exportador de combustíveis renováveis (OSAVA, 2006).

A lei 11.097/05 - também conhecida como Lei do biodiesel - liberou a adição voluntária de $2 \%$ de biodiesel ao diesel distribuído em todo Brasil, até o ano de 2008, quando esse percentual se tornará obrigatório. A demanda de biodiesel para atender a esse percentual é da ordem de 800 milhões de litros por ano, o que, segundo especialistas do governo, equivale a 1,5 milhão de hectares de área cultivada de oleaginosas cerca de $1 \%$ da área produtiva usada pela agricultura brasileira. Atualmente, existem oito usinas de biodiesel credenciadas pela ANP ( Agência Nacional de Petróleo), com capacidade total de produção de aproximadamente 85 milhões de litros por ano. Após a realização de dois leilões, foram vendidos à Petrobras 240 milhões de litros, com preço médio de $\mathrm{R} \$ 1,86 /$ litro, para serem fornecidos até junho de 2007, o que representa aproximadamente $30 \%$ da demanda nacional para a mistura B2 (MARQUES, 2006).

A mesma lei estabelece o percentual obrigatório de $5 \%$ de adição de biodiesel ao diesel a partir de 2013. Este prazo poderá ser reduzido mediante Resolução do CNPE Conselho Nacional do Petróleo, desde que satisfeitas as condições estabelecidas nesta lei (REDE BRASILEIRA DE TECNOLOGIA DE BIODIESEL, 2005).

Ensaios em motores de combustão interna são necessários para a validação do uso das misturas de biodiesel ao diesel mineral. Assim sendo, neste trabalho, objetivou-se avaliar o desempenho comparativo de um motor de ciclo diesel de um trator agrícola alimentado $100 \%$ com diesel e com misturas B2, B5, B20 e B100. Avaliou-se, durante os ensaios, a potência equivalente, o torque equivalente, $\mathrm{o}$ consumo energético e a eficiência térmica do motor.

\section{MATERIAL E MÉTODOS}

Para os ensaios, foi utilizado o motor de ciclo diesel de um trator VALMET 85 id modelo MWM - D225 - 4TVA, de 4 tempos, injeção direta, refrigerado à água, camisas 
úmidas removíveis, com 4 cilindros em linha, cilindrada total de $3778 \mathrm{~cm}^{3}$ e taxa de compressão 18:1, com potência nominal segundo o fabricante de $78 \mathrm{cv}(58,2 \mathrm{~kW})$ a $2300 \mathrm{rpm}$.

O óleo diesel comercial utilizado nos ensaios foi obtido na rede de abastecimento automotiva local, com massa especifica de $0,856 \mathrm{~kg} . \mathrm{L}^{-1}$ a $17,1^{\circ} \mathrm{C}$ e poder calorífico inferior de $41737,00{\mathrm{~kJ} . \mathrm{kg}^{-1}}^{\mathrm{e}}$ o biodiesel utilizado é resultante da transesterificação de óleo de soja, apresentando massa especifica de $0,896 \mathrm{~kg} . \mathrm{L}^{-1}$ a $17,1^{\circ} \mathrm{C}$ e poder calorífico inferior

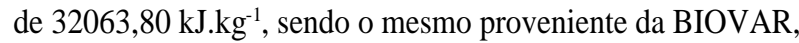
usina de Biodiesel em operação na cidade de Varginha-MG.

$\mathrm{O}$ poder calorífico superior foi determinado no Laboratório de Termodinâmica da Escola de Engenharia de São Carlos (USP - São Carlos), utilizando-se uma bomba calorimétrica IKA 2000 e para a determinação da massa específica de cada mistura de combustíveis utilizou-se uma balança analítica Sartorius-BP221S. Jáo poder calorífico inferior foi determinado segundo a metodologia proposta por Moreira (2006).

Para a medida do consumo de diesel e das misturas de diesel com biodiesel durante os ensaios do motor do trator, foi empregado um medidor volumétrico descrito por Gamero et al. (1986), constando de uma proveta graduada e duas válvulas solenóides que têm a função de controlar o fluxo de entrada e de saída do combustível, permitindo sua medida por diferença de nível em função do tempo.

O dinamômetro utilizado para se obter os valores de torque e potência foi o modelo NEB 200, marca AW DYNAMOMETER, no qual há a transformação da energia mecânica em calor através da utilização de um freio hidráulico, sendo que o calor gerado nesse freio é dissipado pela passagem de água de arrefecimento.

No trabalho em questão não foram avaliadas as condições dos componentes mecânicos internos do motor. A alimentação do motor deu-se em etapas nas quais, em cada uma, foi utilizado óleo diesel comercial e misturas deste com biodiesel nas proporções equivalentes a B2, B5, B20 e B100.

Para a realização dos ensaios do motor, foi adotada a metodologia estabelecida pela norma NBR 5484 da Associação Brasileira de Normas Técnicas (ABNT, 1985), em vigor desde fevereiro de 1985, que fornece os parâmetros relativos ao ensaio dinamométrico de motores do ciclo Otto e Diesel, com as seguintes definições: potência observada $(\mathrm{kW})$, torque (m.N), consumo horário $\left(\mathrm{kg} \cdot \mathrm{h}^{-1}\right)$, etc.

$\mathrm{O}$ torque e a potência do motor foram mensurados por meio da TDP do trator, em regime de plena carga, para as rotações de 700, 600, 540 e $470 \mathrm{rpm}$ da TDP equivalentes às rotações no motor de 2230, 1911, 1720 e 1497 rpm. Foram feitas quatro repetições para cada rotação.

O volume de combustível consumido pelo motor em funcionamento foi obtido pela diferença de nível do combustível na proveta graduada, para um determinado intervalo de tempo de funcionamento do motor. A escala graduada da proveta foi dividida em milímetros e a correspondência volumétrica é de $1,25663 \mathrm{ml} . \mathrm{mm}^{-1}$.

O consumo energético foi calculado em função da massa específica e do poder calorífico inferior de cada combustível ou misturas dos mesmos.

A eficiência térmica foi determinada considerando a potência na TDP e o poder calorífico inferior do combustível para o cálculo da potência teórica, desconsiderando-se as perdas por atrito, conforme Mialhe (1996).

\section{RESULTADOS E DISCUSSÃO}

Os resultados obtidos durante os ensaios dinamométricos podem ser observados na Tabela 1 , destacando-se cada uma das condições de alimentação pré-estabelecidas para o motor.

Como se observa na figura 1 , com o uso de óleo diesel, a curva de potência foi ligeiramente superior, sendo $2,36 \%$ maior que a curva de potência utilizando biodiesel. Esse resultado por si só não representa, em termos de ganho, um valor significativo, inclusive pode até ser explicado por um erro experimental. Entretanto, observase que com as misturas B5 e B20, a potência chegou a superar a potência com diesel em rotações específicas.

A figura 2 demonstra o comportamento do torque, destacando-se os maiores valores para o motor operando com diesel.

O consumo energético, como se observa na figura 3 cresce à medida que se aumenta a rotação do motor, pois, desta forma, exige-se maior potência do motor. À medida que se adiciona biodiesel ao diesel, a quantidade de combustível consumida vai diminuindo, isto acontece em conseqüência do fato de o biodiesel apresentar características de menor poder calorífico que o diesel mineral puro. Ao utilizar o biodiesel puro, o motor apresentou menor consumo energético em relação às demais misturas de combustível e, também, em relação ao diesel mineral puro.

De acordo com a figura 4, a maior eficiência térmica obtida foi ao se usar biodiesel puro e a menor foi ao se utilizar a mistura B2. Esses resultados encontrados, são convergentes com outros publicados em tempos mais remotos, como Goering et al. (1982) e Vinyard et al. (1982). Entretanto, face ao abismo cientifico verificado nesse período em relação ao assunto proposto, esses se tornam relevantes em termos de comparação.

A melhor eficiência térmica foi obtida em torno da rotação do motor equivalente a $540 \mathrm{rpm}$ da TDP, isto é, entre 500 e $600 \mathrm{rpm}$, para todas as misturas de combustíveis. 
Tabela 1 - Ensaio dinamométrico do trator com motor a plena carga para os diferentes combustíveis utilizados (com Potência Reduzida). UFLA, LAVRAS/MG, 2005/2006.

\begin{tabular}{|c|c|c|c|c|c|}
\hline $\begin{array}{c}\text { Rotação } \\
\text { na TDP }\end{array}$ & $\begin{array}{c}\text { Rotação } \\
\text { equivalente } \\
\text { no motor }\end{array}$ & $\begin{array}{c}\text { Potência } \\
\text { Reduzida } \\
\text { na TDP }\end{array}$ & Torque & $\begin{array}{l}\text { Consumo } \\
\text { energético }\end{array}$ & $\begin{array}{c}\text { Eficiência } \\
\text { Térmica }\end{array}$ \\
\hline$(\mathrm{rpm})$ & $(\mathrm{rpm})$ & $(\mathrm{kW})$ & $(\mathrm{m} . \mathrm{N})$ & $\left(\mathrm{kJ} . \mathrm{h}^{-1}\right)$ & $(\%)$ \\
\hline \multicolumn{6}{|c|}{$100 \%$ diesel } \\
\hline 700 & 2230 & 40,25 & 501,50 & 439839,54 & 32,93 \\
\hline 600 & 1911 & 38,79 & 567,00 & 403186,25 & 34,63 \\
\hline 540 & 1720 & 36,65 & 594,75 & 370745,97 & 35,57 \\
\hline 470 & 1497 & 33,70 & 619,75 & 350596,73 & 34,59 \\
\hline \multicolumn{6}{|c|}{$98 \%$ diesel e $2 \%$ biodiesel } \\
\hline 700 & 2230 & 39,70 & 497,25 & 438215,50 & 32,60 \\
\hline 600 & 1911 & 37,75 & 540,75 & 401697,54 & 33,82 \\
\hline 540 & 1720 & 36,12 & 565,50 & 369377,05 & 35,19 \\
\hline 470 & 1497 & 32,82 & 582,00 & 349302,21 & 33,81 \\
\hline \multicolumn{6}{|c|}{$95 \%$ diesel e $5 \%$ biodiesel } \\
\hline 700 & 2230 & 39,81 & 490,5 & 442548,77 & 32,37 \\
\hline 600 & 1911 & 37,80 & 528,75 & 381806,78 & 35,63 \\
\hline 540 & 1720 & 36,84 & 553,25 & 368790,64 & 35,95 \\
\hline 470 & 1497 & 34,53 & 576,00 & 343429,97 & 36,18 \\
\hline \multicolumn{6}{|c|}{$80 \%$ diesel e $20 \%$ biodiesel } \\
\hline 700 & 2230 & 39,76 & 502,25 & 411926,24 & 34,73 \\
\hline 600 & 1911 & 38,77 & 526,75 & 376800,75 & 37,02 \\
\hline 540 & 1720 & 36,78 & 548,00 & 360054,05 & 36,76 \\
\hline 470 & 1497 & 32,79 & 568,00 & 307641,12 & 38,36 \\
\hline \multicolumn{6}{|c|}{$100 \%$ biodiesel } \\
\hline 700 & 2230 & 38,60 & 502,75 & 376469,59 & 36,90 \\
\hline 600 & 1911 & 37,78 & 528,50 & 343603,20 & 39,56 \\
\hline 540 & 1720 & 35,68 & 551,75 & 324299,64 & 39,60 \\
\hline 470 & 1497 & 32,08 & 560,50 & 300652,80 & 38,39 \\
\hline
\end{tabular}

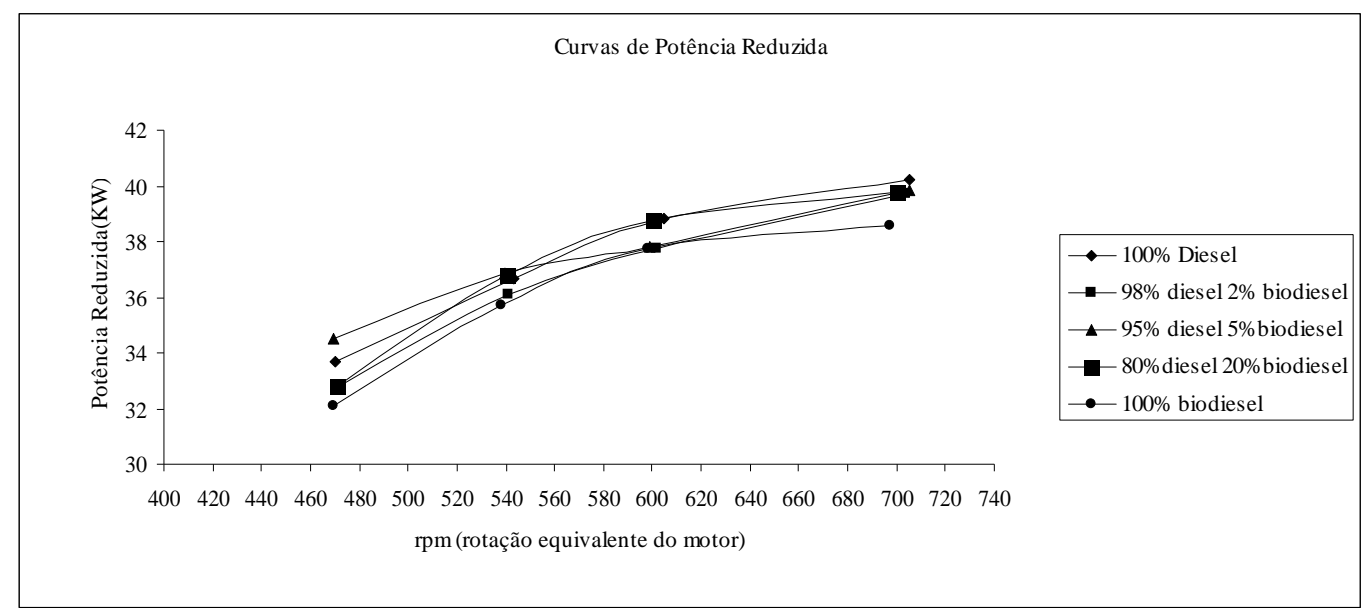

Figura 1 - Curvas de potência do motor do trator a plena carga para os combustíveis utilizados. 


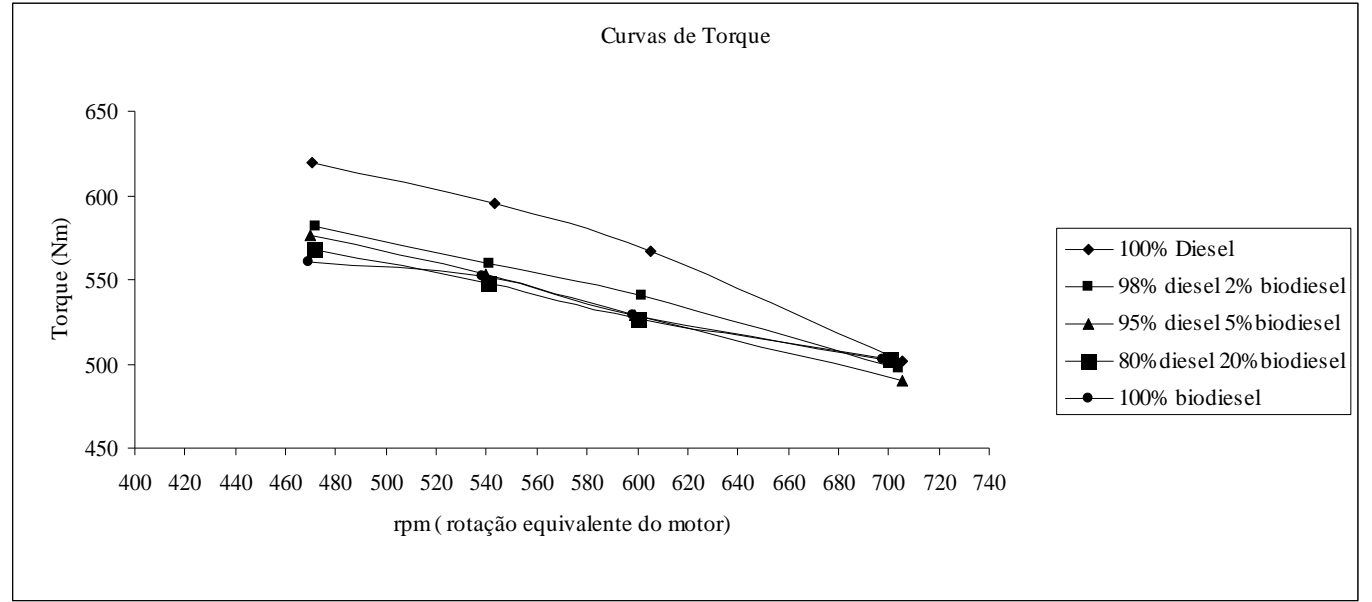

Figura 2 - Curvas de torque do motor do trator a plena carga para os combustíveis utilizados.

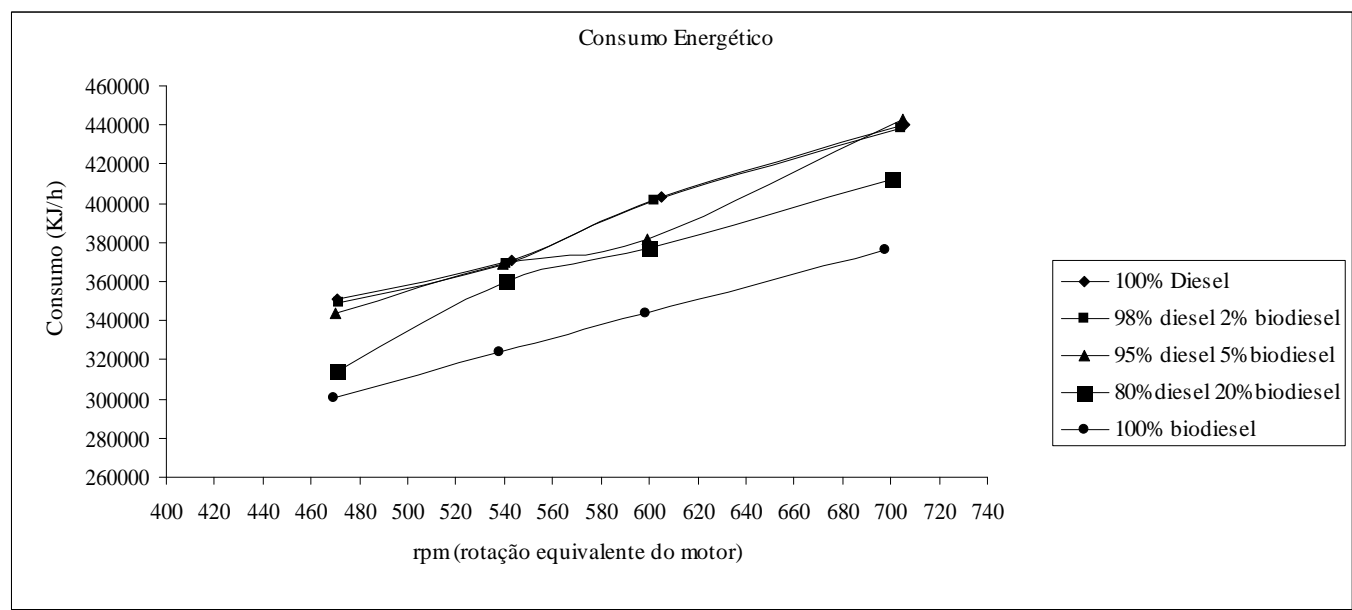

Figura 3 - Curvas de consumo energético do motor do trator a plena carga para os combustíveis utilizados.

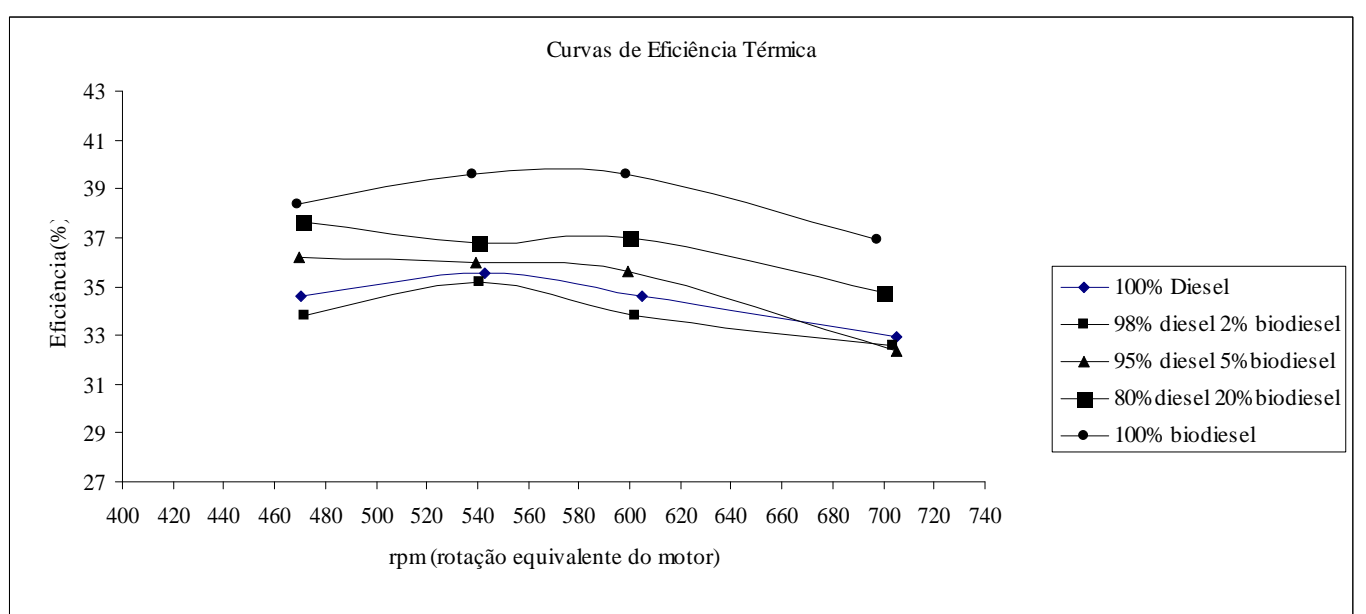

Figura 4 - Curvas de eficiência térmica do motor do trator para os combustíveis utilizados.

Ciênc. agrotec., Lavras, v. 32, n. 5, p. 1588-1593, set./out., 2008 


\section{CONCLUSÕES}

- A potência do motor com o uso de biodiesel em $100 \%$ ou, em misturas com diesel, foi pouco inferior à potência apresentada com o óleo diesel comercial;

- A redução média na potencia do motor observada foi de $1,31 \%$, sendo em alguns regimes de rotação, as misturas B5 e B20 apresentaram potência superior ou igual ao diesel;

- Os valores obtidos de torque quando se utilizou diesel foram em média $7 \%$ maiores do que quando se utilizou $100 \%$ biodiesel;

- A eficiência térmica do motor aumentou quando se usou misturas de biodiesel com diesel, sendo que em média a maior eficiência se observou nas rotações em torno de $540 \mathrm{rpm}$ da TDP equivalente a $1720 \mathrm{rpm}$ do motor;

- Os valores de eficiência térmica ao se utilizar $100 \%$ de biodiesel foram em média $4 \%$ maiores do que quando se utilizou diesel mineral;

- O consumo energético obtido foi diminuindo seus valores nas misturas de combustíveis utilizadas à medida que se aumentava a quantidade de biodiesel misturada ao diesel.

\section{REFERÊNCIAS BIBLIOGRÁFICAS}

ASSOCIAÇÃO BRASILEIRA DE NORMAS TÉCNICAS. Motores alternativos de combustão interna de ignição por compressão (diesel) ou ignição por centelha (Otto) de velocidade angular variável: ensaio: NBR 5484. Rio de Janeiro, 1985.

D’ARCE, M. A. B. R. Grãos e óleos vegetais: matérias primas. Disponível em: <http://Www.esalq.usp.bry. Acesso em: 24 ago. 2005.

GAMERO, C. A.; BENEZ, S. H.; FURLANI JÚNIOR, J. A. Consumo de combustível em diferentes tipos de mobilização do solo. In: CONGRESSO BRASILEIRO DE ENGENHARIA AGRÍCOLA, 15., 1986, Botucatu. Anais... Botucatu: Sociedade Brasileira de Engenharia Agrícola, 1986. p. 1-9.

GOERING, C. E.; CAMPION, R. M.; SCHWAB, A. W.; PRYDE, E. H. Evaluation of soybean oli-aqueous ethanol microemulsions for diesel engines. In: VEGETABLE OIL FUELS: PROCEEDINGS OF THE INTERNATIONAL CONFERENCE ON PLANT AND VEGETABLE OILS AS FUELS, 1982, Michigan. Proceedings... Michigan: American Society of Agricultural Engineers, 1982.
GRANDO, F. A força do combustível verde. Revista do CONFEA, Brasília, v. 9, n. 22, p. 14-17, jun. 2005.

MARQUES, G. G. Biodiesel, bom para o campo e cidade. Informativo da Rede Baiana de Biocombustíveis, Salvador, n. 111, p. 1-2, abr. 2006.

MIALHE, L. G. Máquinas agrícolas: ensaios e certificação. Piracicaba: Fundação de Estudos Agrários Luiz de Queiroz, 1996. $722 \mathrm{p}$.

MOREIRA, A. Combustíveis. São Carlos: USP, 2006. Apostila. Disponível em: <http://Www.netef.eesc.usp.bry. Acesso em: 1 fev. 2006.

OLIVEIRA, L. B.; COSTA, A. O. da. Biodiesel: uma experiência de desenvolvimento sustentável. Disponível em: khttp://Www.biodieselecooleo.com.br/biodieselil estudos>. Acesso em: 25 set. 2005.

OSAVA, M. Arrancam as locomotivas a biodiesel. Disponível em: <ihttp://www.tierramerica.net/2003/1222/, pacentos.shtmil;. Acesso em: 25 abr. 2006.

PARENTE, E. J. de S. Biodiesel: uma aventura tecnológica num país engraçado. Disponível em: <http:// iwww.tecbio.com.bry. Acesso em: 10 fev. 2005.

PROGRAMA NACIONAL DE PRODUÇÃO E USO DE BIODIESEL. Selo combustível social e agricultura familiar.

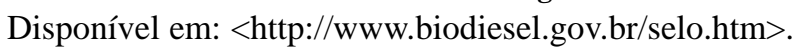
Acesso em: 5 out. 2005 .

REDE BRASILEIRA DE TECNOLOGIA DE BIODIESEL. Testes e ensaios. Disponível em: <http:// LWWw.biodiesel.gov.br/red em: 5 out. 2005 .

VINYARD, S.; RENOLL, E. S.; GOODLING, J. S.; HAWKINS, L.; BUNT, R. C. Properties and performance testing with blends of biomass alcohols, vegetable oils and diesel fuel. In: VEGETABLE OIL FUELS: PROCEEDINGS OF THE INTERNATIONAL CONFERENCE ON PLANT AND VEGETABLE OILS AS FUELS, 1982, Michigan. Proceedings... Michigan: American Society of Agricultural Engineers, 1982. p. 287-293. 